\title{
Case report on anaesthetic management of a patient with self embedding syndrome and difficult airway due to pins in and around trachea
}

\author{
Deepti Dhiman ${ }^{1}$, Divesh Arora ${ }^{1}$
}

\section{Abstract}

Management of a "difficult airway" remains one of the most relevant and challenging tasks for anaesthesiologists. Although practice guidelines and algorithms may help in such situations, yet modifications are required according to the situations. In this case report the anaesthetic management of a patient with self-embedding syndrome having multiple pins inside his trachea and neck has been described. We present our method of successful management in this case and reiterate the role of different methods of correct positioning and intubation in such circumstances. The use of dexmedetomidine helped in achieving spontaneously breathing patient and fiberoptic bronchoscopy used for assessing the tracheal condition before tracheostomy.

Keywords: difficult airway, awake fiberoptic.

\section{Introduction}

Failed or difficult endotracheal intubation and ventilation is a significant cause of morbidity and mortality during anaesthesia [1]. Several methods like the mallampati grading, upper lip bite test and many more have been introduced to identify patients who are in danger of difficult intubation before the initiation of anesthesia [2]. Our patient with multiple pins in and around trachea and neck makes airway management a challenging task for anaesthesiologist. We could not go for mask ventilation and endotracheal intubation straight forward as patient had a pin in pyriform fossa which can damage the cuff. Supraglottic devices could not be used due to this pyriform fossa pin. Due to lack of cooperation on the part of patient awake procedure could not be performed. Techniques for maintaining spontaneous respiration under sedative and analgesic conditions are required. Multiple airway management strategies are present but how we modified the guidelines and used them for patient's benefit is going to be discussed hereby.

We describe a case of patient with self-embedding syndrome with tendency to insert pins inside his body $[3,4]$. Patient even inserted pins in his trachea and this tendency is known as polyembolokoilamania [5]. Sedative medications and fiberscope helped in successfully accomplishing the case.

Written consent was taken from the patient and his relative for the publication of the case.

\section{Case Report}

58 years-old-male patient was admitted under ENT department with complaint of pain in neck with restricted neck movements.
Patient also complained of breathing difficulty and dysphagia but no stridor was present. The patient had one episode of haemoptysis one day before admission. Thorough examination of the patient was done and his X-ray of neck, legs and chest revealed multiple pins (Fig. 1A, 1B). Then the patient underwent whole body CT scan which showed multiple pins in his neck, feet, arms, chest and abdominal wall (Fig. 2). A provisional diagnosis of Self Embedding syndrome was made and decision to remove the pins causing harm to the patient was taken.

Otolaryngologist decided to do indirect laryngoscopy which showed one needle in pyriform fossa with its one end embedded in pharyngeal wall. After bronchoscopy was done by respiratory physicians, 8-10 pins were seen inside the trachea (Fig. 3). Many pins were lying horizontally in the lumen of trachea, a few were present in posterior wall of trachea and puncturing the oesophagus and one pin/needle was present just below the vocal cords making it a difficult airway. Vocal cords were swollen but the movements was normal. In the neck around 100 pins were present therefore decision was taken to remove the pins which were endangering the life of patient. On psychiatric consultation patient was diagnosed to have rare disease called "self-embedding syndrome".

Pre anaesthetic check-up was done day before surgery. The patient was diabetic from past 4 months and was on oral hypoglycaemic drugs. On general physical examination patient had a height of 163 $\mathrm{cm}$ and weight of $62 \mathrm{~kg}$. Patient's heart rate was 90 beats per minute, blood pressure was $110 / 70 \mathrm{mmHg}$, respiratory rate of 20 per minute and room air oxygen saturation was $98 \%$. Airway examination showed mouth opening of $3 \mathrm{~cm}$ and mallampati grade

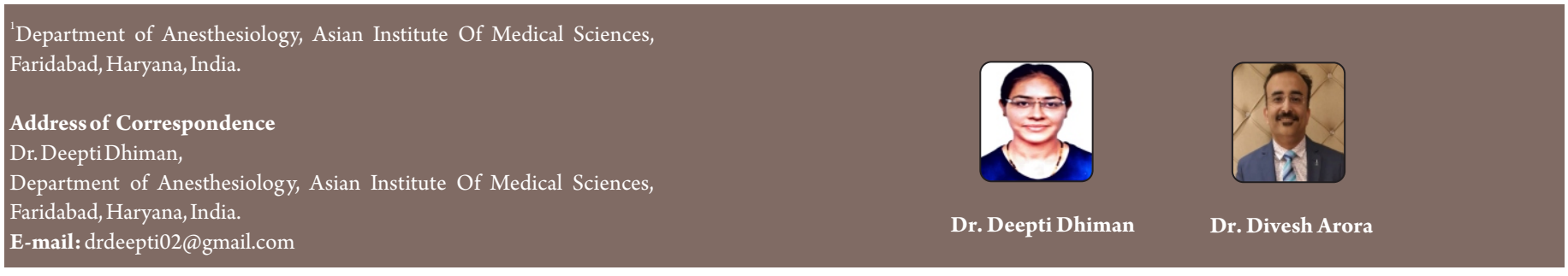

Submitted: 24 June 2020; Reviewed: 1 August 2020; Accepted: 5 February 2021; Published: 10 May 2021

DOI: 10.13107/jaccr.2021.v07i02.174

This is an Open Access article distributed under the terms of the Creative Commons Attribution Non-Commercial-Share Alike 4.0 License (http://creativecommons.org/licenses/by-nc-sa/4.0) which allows others to remix, tweak, and build upon the work non-commercially as long as appropriate credit is given and the new creation are licensed under the identical terms. 


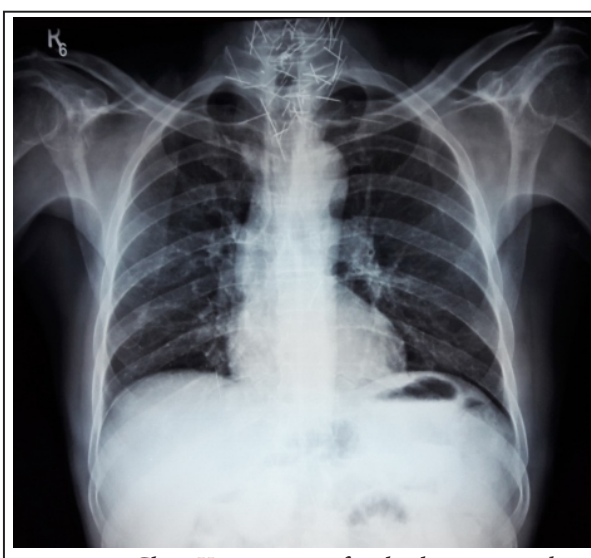

Figure 1A: ChestX-ray image of multiple pins in neck
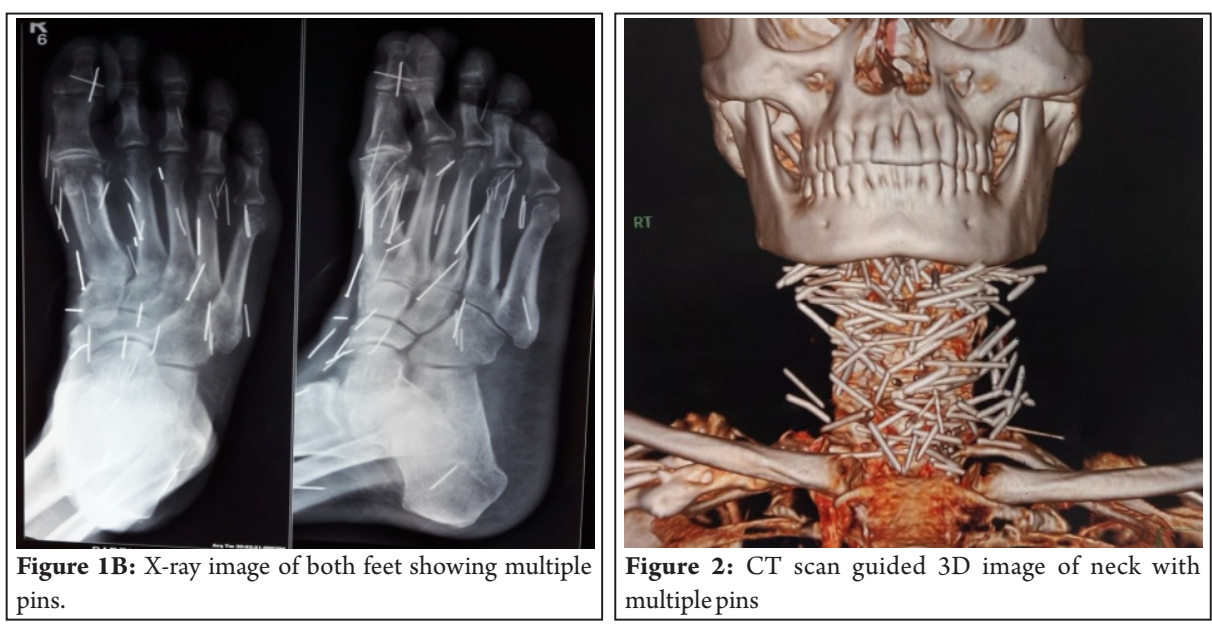

Figure 2: CT scan guided 3D image of neck with multiple pins was 3. Due to multiple pins in neck patient developed fibrosis leading to restricted mobility of neck. All necessary investigations were sent and reports were unremarkable. Blood was cross matched and arranged.

Patient was prepared for the removal of pins in the neck and the big question before our anaesthesia team was "how to plan the anaesthesia?"

After discussing the case with ENT and vascular surgeons it was decided to go for monitored anaesthesia care till the pins in trachea were removed and fibreoptic bronchoscopy would be done so as to monitor removal of pins from trachea.

Informed written consent and high risk consent for postoperative ventilatory support was taken and patient was explained about the eight hours fasting before surgery. Patient was also explained about the procedure of fibreoptic bronchoscopy and tracheostomy. On the day of surgery in preoperative room patient was nebulised with $4 \%$ lignocaine $(4 \mathrm{ml})$ and inj. Glycopyrrolate 40 mcg.kg-1 was given. Operation theatre was prepared with difficult intubation tray including the intubating fibreoptic bronchoscope, cricothyrotomy set and the tracheostomy set.

Patient was shifted to operation theatre and monitored with a noninvasive blood pressure, pulse oximeter, and electrocardiogram. A large bore intravenous assess was taken and balanced salt solution was started. Oxygen supplementation was done with nasal prongs at 4 L.min-1. Inj. Dexmedetomidine loading dose 1mcg.kg-1 over 10 minutes was given followed by infusion at $0.3 \mathrm{mcg} . \mathrm{kg}-1$.hr-1. Inj.
Fentanyl was given in bolus doses of $0.5-1 \mathrm{mcg} . \mathrm{kg}-1$ during the whole procedure up to a total dose of $200 \mathrm{mcg}$. Inj. Dexamethasone was given $8 \mathrm{mg}$ to reduce airway oedema. Patient's vitals were stable. Patient was positioned with neck extension on pillows. Dissection of anterior and carotid triangle of neck was started after infiltrating the neck with local anaesthetic $2 \%$ lignocaine $(5 \mathrm{ml})$ and visualising the pins with the help of $\mathrm{C}$ arm. After clearing the pins around the trachea and exposing the tracheal rings, check fibreoptic bronchoscopy was done and 8-10 pins were still lying in the lumen. Surgeons decided to remove the pin in pyriform fossa first with the help of laryngoscope but failed to visualise it, so Cmac video laryngoscope was used and pin was removed under vision (Fig. 4). Surgeons were finding it difficult to remove the pins lying in the lumen so decision for low tracheostomy was taken to remove the remaining pins through the stoma made in trachea (Fig. 5).

Tracheostomy was made after supplementing sedation with boluses of inj. fentanyl $50 \mathrm{mcg}$ and inj. propofol $100 \mathrm{mg}$. Up till now patient was spontaneously breathing. Armoured endotracheal tube of $8.0 \mathrm{~mm}$ ID was inserted, cuff inflated and air entry was checked. Patient was given inj. vecuronium bromide $5 \mathrm{mg}$ and was ventilated with volume-controlled mode to maintain end tidal $\mathrm{CO}_{2}$ of $35-40$ $\mathrm{mmHg}$. Adequate depth of anaesthesia was achieved with desflurane mac of 0.8 in oxygen with $\mathrm{FiO}_{2}$ of 1 . Before exploring the trachea further patient was ventilated with $\mathrm{FiO}_{2}$ of 1 till we achieved the expiratory oxygen concentration of $>99 \%$. Then endotracheal

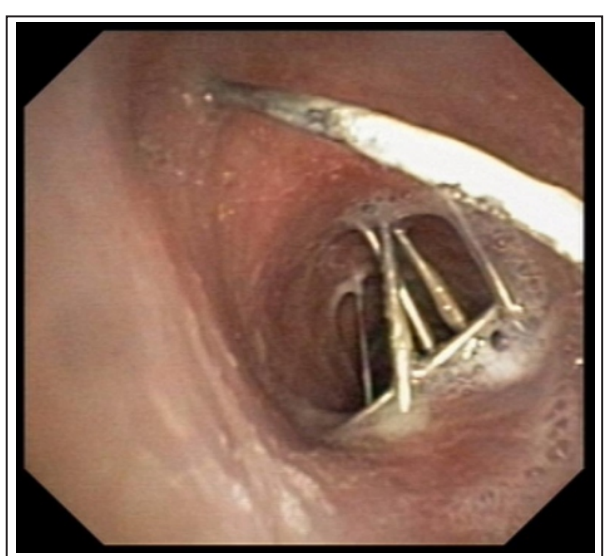

Figure 3: Bronchoscopic image of trachea
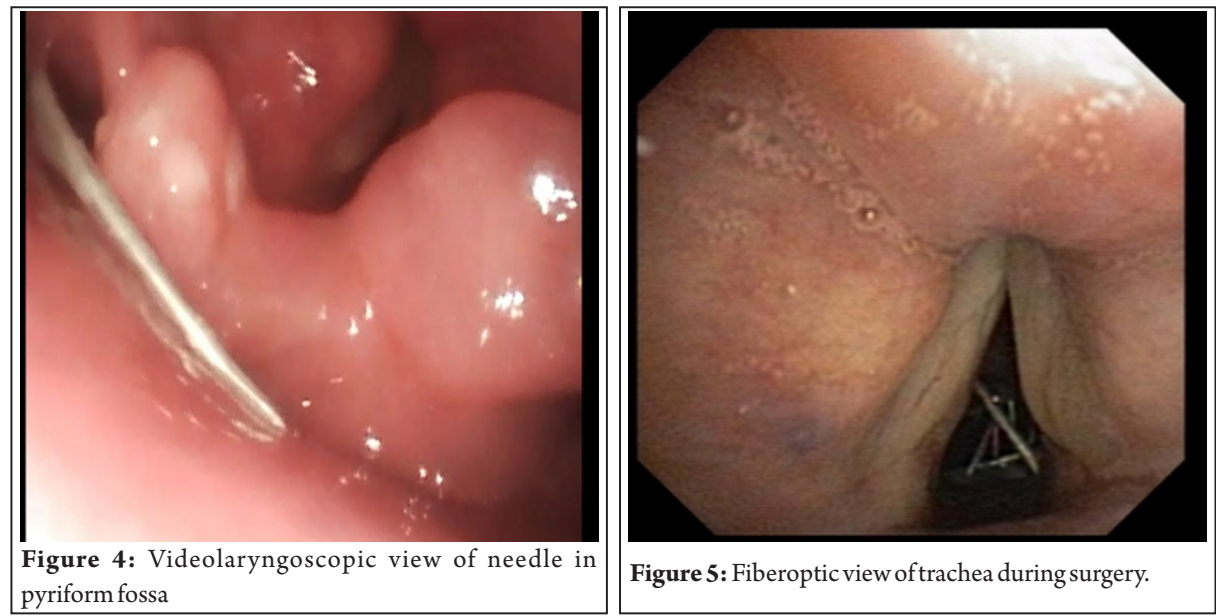

Figure 5: Fiberoptic view of trachea during surgery.

11 | Journal of Anaesthesia and Critical Care Case Reports | Volume 7 | Issue 2 | May-August 2021 | Page 10-13 
tube was removed from the tracheal stoma and trachea was explored. All the remaining 4-5 pins were removed under vision. Endotracheal tube was again inserted and secured with sutures after cuff inflation. Fiberoptic bronchoscopy was done from the mouth to confirm the removal of all pins.

Patient was maintained on desflurane with MAC of 0.8 in oxygen and air with total flow of $500 \mathrm{ml}$ and tidal volume of 5-6ml.kg-1. Neck exploration continued for further 2-3 hours and around 91 pins were removed from neck. Pins which were visualised to pierce the carotids on both the sides were removed by the vascular surgeon and haemostasis was achieved. Many of the pins which were present in the base of tongue were difficult to remove. Few pins were present in the substance of thyroid and in thoracic inlet. These were removed and haemostasis was attained. One unit packed red blood cell was transfused. Inj. Ondansetron $0.15 \mathrm{mg}$.kg1 was given 20 minutes before completion of surgery. At the end of surgery endotracheal tube was replaced with tracheostomy tube of $8.0 \mathrm{~mm}$ internal diameter. Intravenous inj. paracetamol $1 \mathrm{gm}$ and inj. Diclofenac sodium $75 \mathrm{mg}$ were given for analgesia. Neuromuscular blockade was reversed with neostigmine $2.5 \mathrm{mg}$ and glycopyrrolate $0.5 \mathrm{mg}$. When the patient was fully awake and spontaneously breathing, he was shifted to intensive care unit on $\mathrm{T}$ piece with oxygen flow of 3-4 L.min-1. Patient's vitals were stable postoperatively.

\section{Discussion}

Self-embedding of foreign bodies is a form of self-injury which involves the insertion of inanimate objects into soft tissues, under the skin or into muscle, to deliberately hurt them or mutilate their bodies without intending to suicide $[3,4]$. Reasons for selfembedding may be border lying personality disorders, posttraumatic stress disorder, schizophrenia, sadistic behavior. Self embedding is a form of self-mutilation. The consequences of selfembedding can become significant. Wounds can become infected, and this is worsened when foreign objects are inserted deep into the tissue. Infection can extend to bone or muscle, and further, it can damage blood vessels, nerves or tendons. Polyembolokoilamania is inserting a foreign body into one body orifice or more. It may be a manifestation of a psychiatric condition, such as malingering, factitious illness, self-injury, or psychosis [5].

Our patient with multiple pins in and around trachea and neck, with difficult airway was posted for removal of these pins. Preoperative evaluation of the airway is essential in patients with an abnormal airway. Multi-slice CT, which can be reconstituted with three-dimensional (3D) images, can provide a virtual bronchoscopic view [6].

When confronting difficult airway before general anesthesia, the most routine manoeuvre is awake intubation. However, the entire process can be made less distressing by proper patient counselling, topical anesthesia and sedative medications. The commonly used sedatives include propofol, remifentanil, Dexmedetomidine but in India due to non-availability of remifentanil we used propofol with dexmedetomidine. In a study that compared propofol with dexmedetomidine for sedation during fibreoptic nasal intubation, patients in the dexmedetomidine group experienced a lower heartrate response to intubation, showed better tolerance, and had a more stable hemodynamic status [7]. In addition to sedation, fentanyl can be used to relieve pain and blunt nerve reflexes during intubation.

The instruments used in an anticipated difficult airway include a fiberscope and video laryngoscope. In our case, although C-mac laryngoscope could easily have exposed patient's vocal cord, we expected to find it difficult to enter his trachea. Thus only a fiberscope was feasible to obtain a view of the airway in his trachea.

Another approach for a difficult airway is invasive access to enable ventilation before anaesthesia. The American Society of Anaesthesiologists difficult airway algorithm defines invasive access as a surgical or percutaneous airway, jet ventilation and retrograde intubation [8]. However, for our patient, because the pins were located under his vocal cords and entire neck, neither cricothyrotomy nor tracheotomy could be performed before their removal. Vigilance and preparedness is the key to success.

A highly specialized team of ear, nose and throat specialists, anesthesiologists, and operative supporting staff is required in cases of difficult airways or surgeries on the trachea. Careful assessment and operative planning by both the surgeons and anaesthesia team are essential for optimal results [9].

\section{Conclusion}

The anaesthesiologist should have multi-layered contingency plan to handle the airway in difficult airway situations. Even though guidelines are present but they need to be modified according to the situation.

Declaration of patient consent: The authors certify that they have obtained all appropriate patient consent forms. In the form, the patient has given his consent for his images and other clinical information to be reported in the Journal. The patient understands that his name and initials will not be published, and due efforts will be made to conceal his identity, but anonymity cannot be guaranteed.

\section{Conflict of interest: Nil Source of support: None}




\section{References}

1. Benumof JL. Management of the difficult adult airway. With special emphasis on awake tracheal intubation. Anesthesiology. 1991;75(6):1087-1110

2. Mallampati SR, Gatt SP, Gugino LD et al. A clinical sign to predict difficult tracheal intubation: a prospective study. Can Anaesth Soc J. 1985;32(4):429-434.

3. Victor S. Mannarino, Débora C. S. Pereira, Wagner S. Gurgel. SelfEmbedding Behavior in Adults: A Report of Two Cases and a Systematic Review.J Forensic Sci, July 2017;Vol. 62, No. 4.

4. Vidanapathirana M, Gunethilake KMTB. Medico-Legal Significance of 'Self Embedding of Foreign Bodies'. International Journal of Medical Toxicology and Forensic Medicine.2015; 5(3): 171-3.

5. Parulekar SV. Polyembolokoilamania in Gynecology.JPGO.2014;1(3).

6. Toyota K, Uchida H, Ozasa H, Motooka A, Sakura S, Saito Y:
Preoperative airway evaluation using multi-slice three-dimensional computed tomography for a patient with severe tracheal stenosis. Br J Anaesth. 2004;93: 865-867.

7. Tsai CJ, Chu KS, Chen TI, Lu DV, Wang HM, Lu IC: A comparison of the effectiveness of dexmedetomidine versus propofol target-controlled infusion for sedation during fibreoptic nasotracheal intubation. Anaesthesia. 2010;65:254-259.

8. Apfelbaum JL, Hagberg CA, Caplan RA et al American Society of Anesthesiologists Task Force on Management of the Difficult Airway: Practice guidelines for management of the difficult airway: an updated report by the American Society of Anesthesiologists Task Force on Management of the Difficult Airway. Anesthesiology. 2013;118: 251270.

9. Blasberg JD, Wright CD: Surgical considerations in tracheal and carinal resection. Semin Cardiothorac Vasc Anesth. 2012;16: 190-195.

\section{How to Cite this Article}

Dhiman D, Arora D | Case report on anaesthetic management of a patient with self embedding syndrome and difficult airway due to pins in and around trachea $\mid$ Journal of Anaesthesia and Critical Care Case Reports| May-August 2021; 7(2): 10-13. 